\title{
Deconfinement of neutron star matter within the Nambu-Jona-Lasinio model
}

\author{
G. Lugones ${ }^{1}$, A.G. Grunfeld ${ }^{2,3,4}$, N.N. Scoccola ${ }^{2,3,5}$ and C. Villavicencio ${ }^{3}$ \\ 1 Universidade Federal do ABC, Centro de Ciencias Naturais e Humanas, \\ Rua Santa Adélia, 166, 09210-170, Santo André, Brazil \\ 2 CONICET, Rivadavia 1917, (1033) Buenos Aires, Argentina. \\ 3 Departmento de Física, Comisión Nacional de Energía Atómica, (1429) Buenos Aires, Argentina. \\ 4 Department of Physics, Sultan Qaboos University, \\ P.O.Box: 36 Al-Khode 123 Muscat, Sultanate of Oman \\ 5 Universidad Favaloro, Solís 453, (1078) Buenos Aires, Argentina.
}

\begin{abstract}
We study the deconfinement transition of hadronic matter into quark matter under neutron star conditions assuming color and flavor conservation during the transition. We use a two-phase description. For the hadronic phase we use different parameterizations of a non-linear Walecka model which includes the whole baryon octet. For the quark matter phase we use an $S U(3)_{f}$ Nambu-JonaLasinio effective model including color superconductivity. Deconfinement is considered to be a first order phase transition that conserves color and flavor. It gives a short-lived transitory colorlessquark-phase that is not in $\beta$-equilibrium, and decays to a stable configuration in $\tau \sim \tau_{\text {weak }} \sim 10^{-8}$ s. However, in spite of being very short lived, the transition to this intermediate phase determines the onset of the transition inside neutron stars. We find the transition free-energy density for temperatures typical of neutron star interiors. We also find the critical mass above which compact stars should contain a quark core and below which they are safe with respect to a sudden transition to quark matter. Rather independently on the stiffness of the hadronic equation of state (EOS) we find that the critical mass of hadronic stars (without trapped neutrinos) is in the range of $\sim 1.5-$ 1.8 solar masses. This is in coincidence with previous results obtained within the MIT Bag model.
\end{abstract}

PACS numbers: 12.39.Fe, 25.75.Nq, 26.60.Kp

\section{INTRODUCTION}

The core of compact stars can reach densities that are several times larger than the saturation density of nuclear matter. In such extreme conditions the baryons get so compressed that they start to overlap and can produce a deconfined gas of quark matter. An important characteristic of the deconfinement transition in neutron stars, is that just deconfined quark matter is transitorily out of equilibrium with respect to weak interactions. In fact, depending on the temperature, the transition should begin with the quantum or thermal nucleation of a small quark-matter drop near the center of the star. On the other hand, the flavor composition of hadronic matter in $\beta$-equilibrium is different from that of $\beta$-stable quark-matter drop. Roughly speaking, the direct formation of a $\beta$-stable quark-drop with $N$ quarks will need the almost simultaneous conversion of $\sim N / 3$ up and down quarks into strange quarks, a process which is strongly suppressed with respect to the formation of a non $\beta$-stable drop by a factor $\sim G_{\text {Fermi }}^{2 N / 3}$. For typical values of the critical-size $\beta$-stable drop $(N \sim 100-1000$ [1]) the suppression factor is actually tiny. Thus, quark flavor must be conserved during the deconfinement transition [1, 2, 3, 4, 4, 5, 6, 7]. The main consequence of this condition is that the density of the transition is higher than it would be if the direct formation of $\beta$-stable quark-drops were possible. This is easy to understand, since the Gibbs free energy per baryon of $\beta$-equilibrated quark matter is always smaller of that of the non- $\beta$-equilibrated state [6].

Due to the uncertainties in the knowledge of the state of matter at the densities of interest, studies of the deconfinement transition are usually based on the extrapolation to higher densities of an hadronic model valid around the nuclear saturation density $\rho_{0}$, and the extrapolation to $\sim \rho_{0}$ of a quark model that is expected to be valid only for high densities. Within this kind of analysis the (in general) different functional form of both EOSs, induces the phase transition to be first order. Notice that from lattice QCD calculations there are indications that the transition is actually first order in the high-density and low-temperature regime, although this calculations involve temperatures that are still larger than those in neutron stars, and do not include the effect of color superconductivity [8]. In a previous work [6], the deconfinement transition has been analyzed within the frame of the MIT bag model paying special attention to the role of color superconductivity. In the present paper we shall analyze the deconfinement transition employing the Nambu-Jona-Lasinio model in the description of quark matter. For the hadronic phase we shall use a model based on a relativistic Lagrangian of hadrons interacting via the exchange of $\sigma, \rho$, and $\omega$ mesons [9]. For simplicity, the analysis will be made in bulk, i.e. without taking into account the energy cost due to finite size effects in creating a drop of deconfined quark matter in the hadronic environment.

This article is organized as follows. In Sec. [I] we briefly outline the non-linear Walecka model used to describe the hadronic phase. In Sec. III we provide some details of the Nambu-Jona-Lasinio model we employ to describe the 


\begin{tabular}{c|c|c|c|c|c|c|c|c|c}
\hline \hline Label & composition & $x_{\sigma}=x_{\rho}$ & $x_{\omega}$ & $\begin{array}{c}\left(g_{\sigma} / m_{\sigma}\right)^{2} \\
{\left[\mathrm{fm}^{2}\right]}\end{array}$ & $\begin{array}{c}\left(g_{\omega} / m_{\omega}\right)^{2} \\
{\left[\mathrm{fm}^{2}\right]}\end{array}$ & $\begin{array}{c}\left(g_{\rho} / m_{\rho}\right)^{2} \\
{\left[\mathrm{fm}^{2}\right]}\end{array}$ & $b$ & $c$ & $M_{\max }$ \\
\hline GM 1 & baryon octet $+e^{-}$ & 0.6 & 0.653 & 11.79 & 7.149 & 4.411 & 0.002947 & -0.001070 & $1.78 M_{\odot}$ \\
GM 4 & baryon octet $+e^{-}$ & 0.9 & 0.9 & 11.79 & 7.149 & 4.411 & 0.002947 & -0.001070 & $2.2 M_{\odot}$ \\
GM 5 & nucleons $+e^{-}$ & 0.6 & 0.653 & 11.79 & 7.149 & 4.411 & 0.002947 & -0.001070 & $2.35 M_{\odot}$ \\
\hline \hline
\end{tabular}

TABLE I: Parameters of the hadronic equation of state. For each parametrization we give the maximum mass $M_{m a x}$ of a hadronic star.

quark matter phase paying special attention to the conditions of color and flavor conservation. In Sec. IV we present and discuss our numerical results. Finally, in Sec. $\nabla$ a summary with some conclusions is given.

\section{THE HADRONIC PHASE}

For the hadronic phase we shall use a non-linear Walecka model (NLWM) [9, 10, 11] which includes the whole baryon octet. The Lagrangian of the model is given by

$$
\mathcal{L}=\mathcal{L}_{B}+\mathcal{L}_{M}+\mathcal{L}_{L}
$$

where the indices $B, M$ and $L$ refer to baryons, mesons and leptons respectively. For the baryons we have

$$
\mathcal{L}_{B}=\sum_{B} \bar{\psi}_{B}\left[\gamma^{\mu}\left(i \partial_{\mu}-g_{\omega B} \omega_{\mu}-g_{\rho B} \vec{\tau} \cdot \vec{\rho}_{\mu}\right)-\left(m_{B}-g_{\sigma B} \sigma\right)\right] \psi_{B}
$$

with $B$ extending over the nucleons $N=n, p$ and the following hyperons $H=\Lambda, \Sigma^{+}, \Sigma^{0}, \Sigma^{-}, \Xi^{-}$, and $\Xi^{0}$. The contribution of the mesons $\sigma, \omega$ and $\rho$ is given by

$$
\begin{aligned}
\mathcal{L}_{M}= & \frac{1}{2}\left(\partial_{\mu} \sigma \partial^{\mu} \sigma-m_{\sigma}^{2} \sigma^{2}\right)-\frac{b}{3} m_{N}\left(g_{\sigma} \sigma\right)^{3}-\frac{c}{4}\left(g_{\sigma} \sigma\right)^{4} \\
& -\frac{1}{4} \omega_{\mu \nu} \omega^{\mu \nu}+\frac{1}{2} m_{\omega}^{2} \omega_{\mu} \omega^{\mu}-\frac{1}{4} \vec{\rho}_{\mu \nu} \cdot \vec{\rho}^{\mu \nu}+\frac{1}{2} m_{\rho}^{2} \vec{\rho}_{\mu} \cdot \vec{\rho}^{\mu},
\end{aligned}
$$

where the coupling constants are

$$
g_{\sigma B}=x_{\sigma B} g_{\sigma}, g_{\omega B}=x_{\omega B} g_{\omega}, \quad g_{\rho B}=x_{\rho B} g_{\rho}
$$

where $x_{\sigma B}, x_{\omega B}$ and $x_{\rho B}$ are equal to 1 for the nucleons and acquire different values for the other baryons depending on the parametrization (see below). The leptonic sector is included as a free Fermi gas which does not interact with the hadrons, i.e.

$$
\mathcal{L}_{L}=\sum_{l} \bar{\psi}_{l}\left(i \not \partial-m_{l}\right) \psi_{l}
$$

There are five constants in the model that are determined by the properties of nuclear matter, three that determine the nucleon couplings to the scalar, vector and vector-isovector mesons $g_{\sigma} / m_{\sigma}, g_{\omega} / m_{\omega}, g_{\rho} / m_{\rho}$, and two that determine the scalar self interactions $b$ and $c$. It is assumed that all hyperons in the octet have the same coupling than the $\Lambda$. These couplings are expressed as a ratio to the nucleon couplings mentioned above, that we thus simply denote $x_{\sigma}, x_{\omega}$ and $x_{\rho}$. In the present work we use three parameterizations for the constants. One of them is the standard parameterization GM1 given by Glendenning-Moszkowski [9], as shown in Table I. This parametrization employs "low" values for $x_{\sigma}, x_{\omega}$ and $x_{\rho}$. Larger values of these couplings make the EOS stiffer and increase the value of the maximum mass of hadronic stars to values above than $2 M_{\odot}$, see Table I.

\section{THE QUARK PHASE}

In order to study the just deconfined quark matter phase we use an $S U(3)_{f}$ NJL effective model which also includes color superconducting quark-quark interactions. The corresponding lagrangian is given by

$$
\mathcal{L}=\bar{\psi}(i \not \partial-\hat{m}) \psi+G \sum_{a=0}^{8}\left[\left(\bar{\psi} \tau_{a} \psi\right)^{2}+\left(\bar{\psi} i \gamma_{5} \tau_{a} \psi\right)^{2}\right]
$$




$$
+2 H \sum_{A, A^{\prime}=2,5,7}\left[\left(\bar{\psi} i \gamma_{5} \tau_{A} \lambda_{A^{\prime}} \psi_{C}\right)\left(\bar{\psi}_{C} i \gamma_{5} \tau_{A} \lambda_{A^{\prime}} \psi\right)\right]
$$

where $\hat{m}=\operatorname{diag}\left(m_{u}, m_{d}, m_{s}\right)$ is the current mass matrix in flavor space. The matrices, $\tau_{i}$ and $\lambda_{i}$ with $i=1, . ., 8$ are the Gell-Mann matrices corresponding to the flavor and color groups respectively, and $\tau_{0}=\sqrt{2 / 3} 1_{f}$. Finally, the charge conjugate spinors are defined as follows: $\psi_{C}=C \bar{\psi}^{T}$ and $\bar{\psi}_{C}=\psi^{T} C$, where $\bar{\psi}=\psi^{\dagger} \gamma^{0}$ is the Dirac conjugate spinor and $C=i \gamma^{2} \gamma^{0}$.

The lagrangian in Eq. (6) leads to local chirally invariant current-current interactions in the quark-antiquark and quark-quark channels. The latter is expected to be responsible for the presence of a color-superconducting phase in the region of low temperatures and moderate chemical potentials. Here for simplicity we do not include flavor mixing effects. The values of the quark masses and the coupling constant $G$ can be obtained from the meson properties in the vacuum. On the other hand, an estimate of $H / G$ can be obtained from Fierz transformation of the one-gluon-exchange interactions in which case one gets $H / G=0.75$, which is the value we will use hereafter.

To be able to determine the relevant thermodynamical quantities we have to obtain the grand canonical thermodynamical potential $\Omega\left(T, \mu_{f c}\right)$ at finite temperature $T$ and chemical potentials $\mu_{f c}$. Here, $f=(u, d, s)$ and $c=(r, g, b)$ denote flavor and color indices respectively. For this purpose we start from Eq. (6) and perform the usual bosonization of the theory. This can be done by introducing scalar and pseudoscalar meson fields $\sigma_{a}$ and $\pi_{a}$ respectively, together with the bosonic diquark field $\Delta_{A}$. In this work we consider the quantities obtained within the mean field approximation. Thus, we only keep the non-vanishing vacuum expectation values of these fields and drop the corresponding fluctuations. For the meson fields this implies $\hat{\sigma}=\sigma_{a} \tau_{a}=\operatorname{diag}\left(\sigma_{u}, \sigma_{d}, \sigma_{s}\right)$ and $\pi_{a}=0$. Concerning the diquark mean field, we will assume that in the density region of interest only the 2SC phase might be relevant. Thus, we adopt the ansatz $\Delta_{5}=\Delta_{7}=0, \Delta_{2}=\Delta$. Integrating out the quark fields and working in the framework of the Matsubara and Nambu-Gorkov formalism we obtain

$$
\Omega_{\mathrm{MFA}}\left(T, \mu_{f c}, \sigma_{f},|\Delta|\right)=-\frac{T}{2} \sum_{n=-\infty}^{\infty} \int \frac{d^{3} k}{(2 \pi)^{3}} \ln \operatorname{det}\left[\frac{S^{-1}(k)}{T}\right]+\frac{1}{4 G}\left(\sigma_{u}^{2}+\sigma_{d}^{2}+\sigma_{s}^{2}\right)+\frac{|\Delta|^{2}}{2 H}
$$

where

$$
S^{-1}=\left(\begin{array}{cc}
\not k-\hat{M}+\gamma_{0} \hat{\mu} & -\Delta \gamma_{5} \tau_{2} \lambda_{2} \\
\Delta^{*} \gamma_{5} \tau_{2} \lambda_{2} & \not k-\hat{M}-\gamma_{0} \hat{\mu}
\end{array}\right) .
$$

Here, we have used $k=((2 n+1) \pi T, \vec{k}), \hat{M}=\operatorname{diag}\left(M_{u}, M_{d}, M_{s}\right)$ with $M_{f}=m_{f}+\sigma_{f}$, and $\hat{\mu}=\operatorname{diag}\left(\left\{\mu_{f c}\right\}\right)$ in flavor $\otimes$ color space. The determinant of this $72 \times 72$ matrix can be calculated analytically if $M_{u}=M_{d}$. Thus, in what follows we will use the approximation $m_{u}=m_{d} \equiv m$ and $\sigma_{u}=\sigma_{d} \equiv \sigma$ which implies $M_{u}=M_{d} \equiv M$. A detailed procedure for the calculation of the determinant can be found in Ref. [12, 13, 14]. The resulting contribution to the thermodynamical potential is

$$
-\frac{T}{2} \sum_{n} \int \frac{d^{3} k}{(2 \pi)^{3}} \ln \operatorname{det}\left[S^{-1}(k) / T\right]=2 \int^{\Lambda} \frac{d^{3} k}{(2 \pi)^{3}} \sum_{\alpha=1}^{9} \omega\left(x_{i}, y_{i}\right)+\text { constant }
$$

where $\Lambda$ is the cut-off of the theory and $\omega(x, y)$ is defined by

$$
\omega(x, y)=-\left[x+T \ln \left[1+e^{-(x-y) / T}\right]+T \ln \left[1+e^{-(x+y) / T}\right]\right],
$$

with

$$
\begin{gathered}
x_{1,2}=E, \quad x_{3,4,5}=E_{s}, \quad x_{6,7}=\sqrt{\left[E+\frac{\left(\mu_{u r} \pm \mu_{d g}\right)}{2}\right]^{2}+\Delta^{2}}, \quad x_{8,9}=\sqrt{\left[E+\frac{\left(\mu_{u g} \pm \mu_{d r}\right)}{2}\right]^{2}+\Delta^{2}} \\
y_{1}=\mu_{u b}, \quad y_{2}=\mu_{d b}, \quad y_{3}=\mu_{s r}, \quad y_{4}=\mu_{s g}, \quad y_{5}=\mu_{s b}, \quad y_{6,7}=\frac{\mu_{u r}-\mu_{d g}}{2}, \quad y_{8,9}=\frac{\mu_{u g}-\mu_{d r}}{2}
\end{gathered}
$$

where $E=\sqrt{\vec{k}^{2}+M^{2}}, E_{s}=\sqrt{\vec{k}^{2}+M_{s}^{2}}$. 


\begin{tabular}{l|ccccc}
\hline \hline & $m_{u, d}[\mathrm{Mev}]$ & $m_{s}[\mathrm{Mev}]$ & $\Lambda[\mathrm{Mev}]$ & $G \Lambda^{2}$ & $H / G$ \\
\hline set 1 & 5.5 & 112.0 & 602.3 & 4.638 & $3 / 4$ \\
set 2 & 5.5 & 110.05 & 631.4 & 4.370 & $3 / 4$ \\
\hline
\end{tabular}

TABLE II: The two sets of NJL parameters.

Finally, we include the thermodynamical potential for non-interacting ultra-relativistic electrons

$$
\Omega_{e}=-\frac{\mu_{e}^{4}}{12 \pi^{2}}-\frac{\mu_{e}^{2} T^{2}}{6}-\frac{7 \pi^{2} T^{4}}{180}
$$

where $\mu_{e}$ is the electron chemical potential. Therefore, the total potential for the quark matter electron system is

$$
\Omega\left(T,\left\{\mu_{f c}\right\}, \mu_{e}, \sigma, \sigma_{s}\right)=\frac{1}{\pi^{2}} \int_{0}^{\Lambda} d k k^{2} \sum_{i} \omega\left(x_{i}, y_{i}\right)+\frac{1}{4 G}\left(\sigma_{u}^{2}+\sigma_{d}^{2}+\sigma_{s}^{2}\right)+\frac{|\Delta|^{2}}{2 H}-\Omega_{\mathrm{vac}}+\Omega_{e} .
$$

Here we have subtracted the constant $\Omega_{\text {vac }}$ in order to have a vanishing pressure at vanishing temperature and chemical potentials. From the grand thermodynamic potential $\Omega$ we can readily obtain the pressure $P=-\Omega$, the number density of quarks of each flavor and color

$$
n_{f c}=-\frac{\partial \Omega}{\partial \mu_{f c}}=-\frac{1}{\pi^{2}} \int_{0}^{\Lambda} d k k^{2} \frac{\partial}{\partial \mu_{f c}}\left(\sum_{i} \omega\left(x_{i}, y_{i}\right)\right)
$$

and the number density of electrons

$$
n_{e}=-\frac{\partial \Omega}{\partial \mu_{e}}
$$

The corresponding number densities of each flavor, $n_{f}$, and of each color, $n_{c}$, in the quark phase are given by

$$
n_{f}=\sum_{c} n_{f c} \quad, \quad n_{c}=\sum_{f} n_{f c} .
$$

Finally, the baryon number density $n_{B}$ reads

$$
n_{B}=\frac{1}{3} \sum_{f c} n_{f c}=\frac{1}{3}\left(n_{u}+n_{d}+n_{s}\right)
$$

and the Gibbs free energy per baryon is

$$
g_{\mathrm{quark}}=\frac{1}{n_{B}}\left(\sum_{f c} \mu_{f c} n_{f c}+\mu_{e} n_{e}\right) .
$$

For the NJL model we use two sets of parameters. They have been derived from those used in refs. [15] and [16] by neglecting the 't Hooft interaction. To do that we have followed the procedure proposed in 17. Namely, keeping $\Lambda$ and $m$ fixed we have varied $G$ and $m_{s}$ in order to obtain $M=367.6 \mathrm{MeV}$ and $M_{s}=549.5 \mathrm{MeV}$ at zero temperature and density. The resulting parameter sets are given in Table 1 .

In order to derive a quark matter EOS from the above formulae it is necessary to impose a suitable number of conditions on the variables $\left\{\mu_{f c}\right\}, \mu_{e}, \sigma, \sigma_{s}$ and $\Delta$. Three of these conditions are consequences from the fact that the thermodynamically consistent solutions correspond to the stationary points of $\Omega$ with respect to $\sigma, \sigma_{s}$, and $\Delta$. Thus, we have

$$
\partial \Omega / \partial \sigma=0 \quad, \quad \partial \Omega / \partial \sigma_{s}=0 \quad, \quad \partial \Omega / \partial|\Delta|=0
$$

To obtain the remaining conditions one must specify the physical situation in which one is interested in. In many astrophysical applications considered in the literature quark matter in $\beta$-equilibrium was analyzed. In such a case chemical equilibrium is maintained by weak interactions among quarks, e.g. $d \leftrightarrow u+e^{-}+\bar{\nu}_{e}, s \leftrightarrow u+e^{-}+\bar{\nu}_{e}, u+d \leftrightarrow$ $u+s$. Moreover, for neutron stars older than a few minutes, neutrinos can leave the system. Thus, lepton number is 
not conserved and we have four independent conserved charges, namely the electric charge $n_{Q}=\frac{2}{3} n_{u}-\frac{1}{3} n_{d}-\frac{1}{3} n_{s}-n_{e}$ and the three color charges $n_{u}, n_{d}$ and $n_{s}$. Instead of $n_{u}, n_{d}$ and $n_{s}$, the linear combinations $n=n_{r}+n_{g}+n_{b}$, $n_{3}=n_{r}-n_{g}$ and $n_{8}=\frac{1}{\sqrt{3}}\left(n_{r}+n_{g}-2 n_{b}\right)$ are often used. Here $n$ is the total quark number density (i.e. $\left.n=3 n_{B}\right)$ and $n_{3}$ and $n_{8}$ describe color asymmetries. The four conserved charges $\left\{n_{j}\right\}=\left\{n, n_{3}, n_{8}, n_{Q}\right\}$ are related to four independent chemical potentials $\left\{\mu_{j}\right\}=\left\{\mu, \mu_{3}, \mu_{8}, \mu_{Q}\right\}$ such that $n_{j}=-\partial \Omega / \partial \mu_{j}$. The individual quark chemical potentials $\mu_{f c}$ are given by

$$
\mu_{f c}=\mu+\mu_{Q}\left[\frac{1}{2}\left(\tau_{3}\right)_{f f}+\frac{1}{2 \sqrt{3}}\left(\tau_{8}\right)_{f f}\right]+\mu_{3}\left(\lambda_{3}\right)_{c c}+\mu_{8}\left(\lambda_{8}\right)_{c c}
$$

where, as before, $\tau_{i}$ and $\lambda_{i}$ are the Gell-Mann matrices in flavor and color space respectively. The electron chemical potential is $\mu_{e}=-\mu_{Q}$, thus we have

$$
\mu_{d c}=\mu_{s c}=\mu_{u c}+\mu_{e}
$$

for all colors $c$, which are the $\beta$-equilibrium conditions. For electrically and color neutral matter we have also the conditions:

$$
n_{Q} \equiv-\frac{\partial \Omega}{\partial \mu_{Q}}=0 \quad, \quad n_{3} \equiv-\frac{\partial \Omega}{\partial \mu_{3}}=0 \quad, \quad n_{8} \equiv-\frac{\partial \Omega}{\partial \mu_{8}}=0
$$

Employing the above conditions, the system can be characterized by two independent variables, e.g. $\left(T, n_{B}\right)$ or $(T, P)$.

The conditions given in Eqs.(2122) are extensively employed to describe quark matter in $\beta$-equilibrium. However, in this paper we deal with just deconfined quark matter which is temporarily out of $\beta$-equilibrium. As already emphasized in [1, 2, 3, 4, 5, 6, 7] the appropriate condition in this case is flavor conservation between hadronic and deconfined quark matter. This can be written as

$$
Y_{f}^{H}=Y_{f}^{Q} \quad f=u, d, s, L
$$

being $Y_{f}^{H} \equiv n_{f}^{H} / n_{B}^{H}$ and $Y_{i}^{Q} \equiv n_{f}^{Q} / n_{B}^{Q}$ the abundances of each particle in the hadron and quark phase respectively. In other words, the just deconfined quark phase must have the same "flavor" composition than the $\beta$-stable hadronic phase from which it has been originated. Notice that, since the hadronic phase is assumed to be electrically neutral, flavor conservation ensures automatically the charge neutrality of the just deconfined quark phase.

The conditions given in Eq. (23) can be re-written as follows

$$
\begin{gathered}
n_{d}=\xi n_{u}, \\
n_{s}=\eta n_{u}, \\
3 n_{e}=2 n_{u}-n_{d}-n_{s},
\end{gathered}
$$

where $n_{i}$ is the particle number density of the $i$-species in the quark phase. The quantities $\xi \equiv Y_{d}^{H} / Y_{u}^{H}$ and $\eta \equiv Y_{s}^{H} / Y_{u}^{H}$ are functions of the pressure and temperature, and they characterize the composition of the hadronic phase. These expressions are valid for any hadronic EOS. For hadronic matter containing $n, p, \Lambda, \Sigma^{+}, \Sigma^{0}, \Sigma^{-}, \Xi^{-}$ and $\Xi^{0}$, we have

$$
\begin{aligned}
\xi & =\frac{n_{p}+2 n_{n}+n_{\Lambda}+n_{\Sigma^{0}}+2 n_{\Sigma^{-}}+n_{\Xi^{-}}}{2 n_{p}+n_{n}+n_{\Lambda}+2 n_{\Sigma^{+}}+n_{\Sigma^{0}}+n_{\Xi^{0}}}, \\
\eta & =\frac{n_{\Lambda}+n_{\Sigma^{+}}+n_{\Sigma^{0}}+n_{\Sigma^{-}}+2 n_{\Xi^{0}}+2 n_{\Xi^{-}}}{2 n_{p}+n_{n}+n_{\Lambda}+2 n_{\Sigma^{+}}+n_{\Sigma^{0}}+n_{\Xi^{0}}} .
\end{aligned}
$$

Additionally, the deconfined phase must be locally colorless; thus it must be composed by an equal number of red, green and blue quarks

$$
n_{r}=n_{g}=n_{b}
$$

Also, $u r, u g, d r$, and $d g$ pairing will happen provided that $|\Delta|$ is nonzero, leading to

$$
n_{u r}=n_{d g}, \quad n_{u g}=n_{d r}
$$


In order to have all Fermi levels at the same value, we consider [6]

$$
\begin{aligned}
n_{u g} & =n_{u r} \\
n_{s b} & =n_{s r} .
\end{aligned}
$$

These two equations, together with Eqs. (29) and (30) imply that $n_{u r}=n_{u g}=n_{d r}=n_{d g}$ and $n_{s r}=n_{s g}=n_{s b}$.

Finally, including the conditions given in Eq.(19) we have 12 equations involving the 13 unknowns $\left(\sigma, \sigma_{s},|\Delta|, \mu_{e}\right.$ and $\left.\left\{\mu_{f c}\right\}\right)$. For given value of one of the chemical potentials (e.g. $\mu_{u r}$ ), the set of equations can be solved once the values of the parameters $\xi, \eta$ and the temperature $T$ are given. Instead of $\mu_{u r}$, we can provide a value of the Gibbs free energy per baryon $g_{\text {quark }}$ or the pressure $P$ and solve simultaneously Eqs. (24)-(32) together with Eq. (19) in order to obtain $\sigma, \sigma_{s},|\Delta|, \mu_{e}$ and $\left\{\mu_{f c}\right\}$.

The above conditions represent a state that fulfills all the physical requirements of the just deconfined phase, i.e. it is color and electrically neutral, and it has a "flavor content" determined by the parameters $\xi$ and $\eta$ (both related to the hadronic phase through Eqs. (27) and (28)).

The set of twelve equations can be summarized as follows [ $[\underline{6}]$

$$
\begin{gathered}
n_{u b}=2 \frac{2-\xi}{1+\xi} n_{u r}, \quad n_{d b}=2 \frac{2 \xi-1}{1+\xi} n_{u r}, \quad n_{s r}=n_{s g}=n_{s b}=\frac{2 \eta}{1+\xi} n_{u r}, \\
n_{u g}=n_{d r}=n_{d g}=n_{u r}, \quad n_{e}=\frac{2(2-\xi-\eta)}{1+\xi} n_{u r},
\end{gathered}
$$

together with equation (19).

\section{DECONFINEMENT TRANSITION IN NEUTRON STAR MATTER}

For simplicity, the analysis that follows will be made in bulk, i.e. without taking into account the energy cost due to finite size effects in creating a drop of deconfined quark matter in the hadronic environment. Finite size effects on the nucleation of color superconducting quark bubbles in a cold deleptonized hadronic medium have been recently analyzed employing the MIT bag model in the description of the quark phase 7]. As a consequence of the surface effects, it is necessary to have an overpressure with respect to the bulk transition point. However, since this effect is not very large we leave its consideration for a future more detailed work.

In order to determine the transition conditions, we apply the Gibbs criteria, i.e. we assume that deconfinement will occur when the pressure and Gibbs energy per baryon are the same for both hadronic matter and quark matter at a given common temperature. Thus, we have

$$
g_{h}=g_{q} \quad, \quad P_{h}=P_{q} \quad, \quad T_{h}=T_{q},
$$

where the index $h$ refers to hadron matter and the index $q$ to quark matter. The results are displayed in Fig. 1 where we show the Gibbs free energy per baryon $g$ at the transition point as a function of the temperature. The hadronic phase is described by the three parameterizations of the EOS given in Sec. II (see Table I). For the quark phase we adopt the NJL model described above (using flavor conservation conditions) with the two parameterizations given in Table II. If hadronic matter has a temperature $T_{h}$ and a Gibbs free energy per baryon $g_{h}$ lying to the left of a given curve, then the deconfinement transition is not possible for that parameterizations of the EOSs. In the right side region of a given curve the preferred phase is deconfined quark matter.

Let us now examine the consequences of the above results on the structure of compact stars. Stars containing quark phases fall into two main classes: hybrid stars (where quark matter is restricted to the core) and strange stars (made up completely by quark matter). It is expected that both kinds of stars cannot exist simultaneously in Nature, but it is not know which one would be realized (if any). This depends on whether the energy per baryon of $\beta$-equilibrated quark matter at zero pressure and zero temperature is less than the neutron mass (the so called "absolute stability" condition [18]). Analysis made within the MIT bag model shows that there is a room in the parameter space for the existence of strange stars. Moreover, pairing enlarges substantially the region of the parameter space where $\beta$-stable quark matter has an energy per baryon smaller than the neutron mass [19, 20]. Although the gap effect does not dominate the energetics, being of the order $(\Delta / \mu)^{2} \sim$ a few percent, the effect is substantially large near the zeropressure point (which determines the stability and also the properties of the outer layers and surface of the star). As a consequence, a "CFL strange matter" is allowed for the same parameters that would otherwise produce unbound strange matter without pairing [19]. However, within the NJL model, the strange matter hypothesis is not favored, 


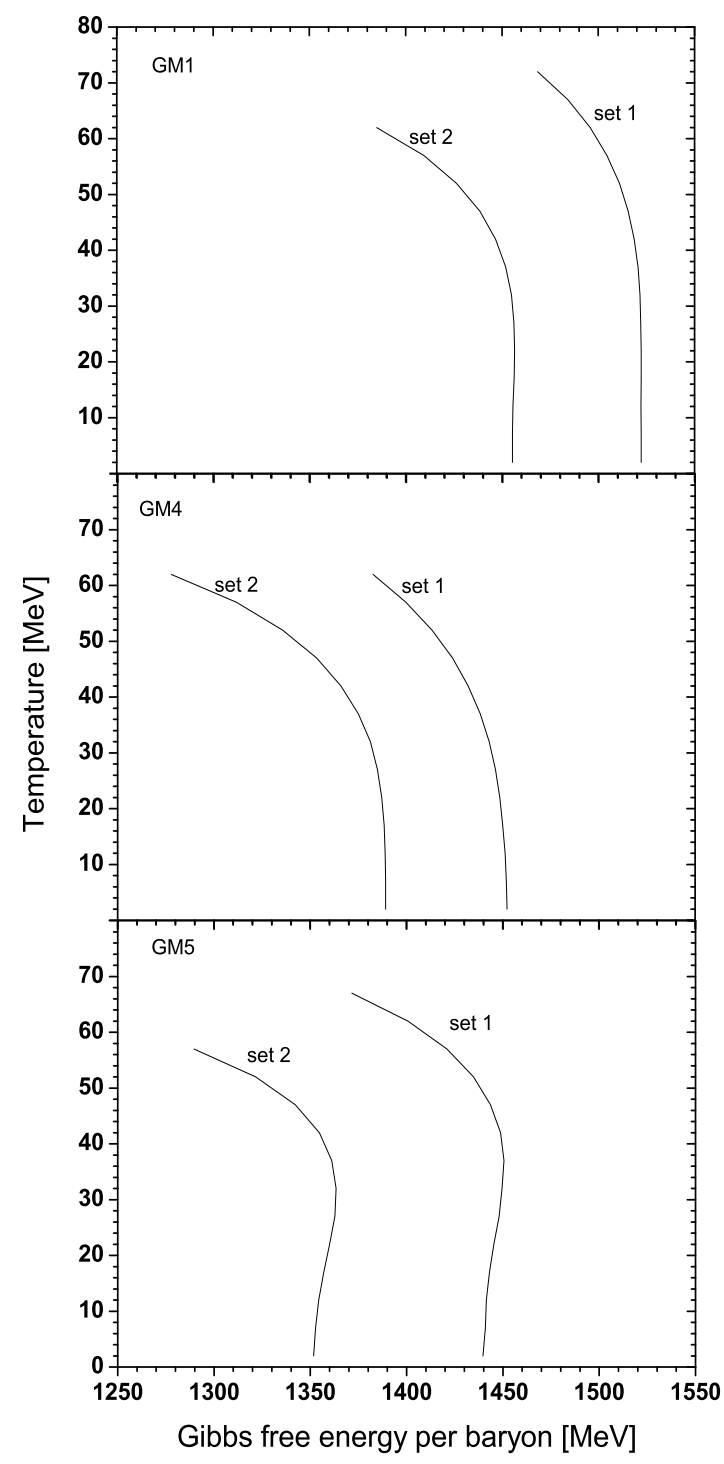

FIG. 1: For each temperature we show the Gibbs free energy density per barion $g$ of the deconfinement phase transition. The hadronic phase is described by the GM1, GM4 and GM5 parametrization of the EOS given in Sec. II (see Table I). For the quark phase we adopt the two parameterizations of the NJL model given in Table II. If hadronic matter has a temperature $T_{h}$ and a Gibbs free energy density per barion $g_{h}$ lying to the left of a given curve, then the deconfinement transition is not possible for that parameterizations of the EOSs. If the point $\left(g_{h}, T_{h}\right)$ lies to the right of the curve the preferred phase is deconfined quark matter.

at least for the most accepted parameterizations of the EOS [17]. Thus, stars containing quark phases are believed to be hybrid stars within the NJL model.

At a given temperature there is a univocal relation between the mass of a compact star and its central pressure (or equivalently the Gibbs free energy per barion at the center of the star). Thus, we can employ the results given in Fig. 1 to calculate the critical compact star mass $M_{c r}$ above which they should contain a quark core. With this purpose, we integrate the Tolman-Oppenheimer-Volkoff equations of relativistic stellar structure employing the hadronic EOSs given in Table I, and identify the mass of the pure hadronic star for which the Gibbs free energy per barion $g_{h}$ at the center is equal to the critical $g$ given in Fig. 1. This is called critical mass $M_{c r}$ because pure hadronic stars with $M_{h}>M_{c r}$ are very unlikely to be observed, while pure hadronic stars with $M_{h}<M_{c r}$ are safe with respect to a sudden 


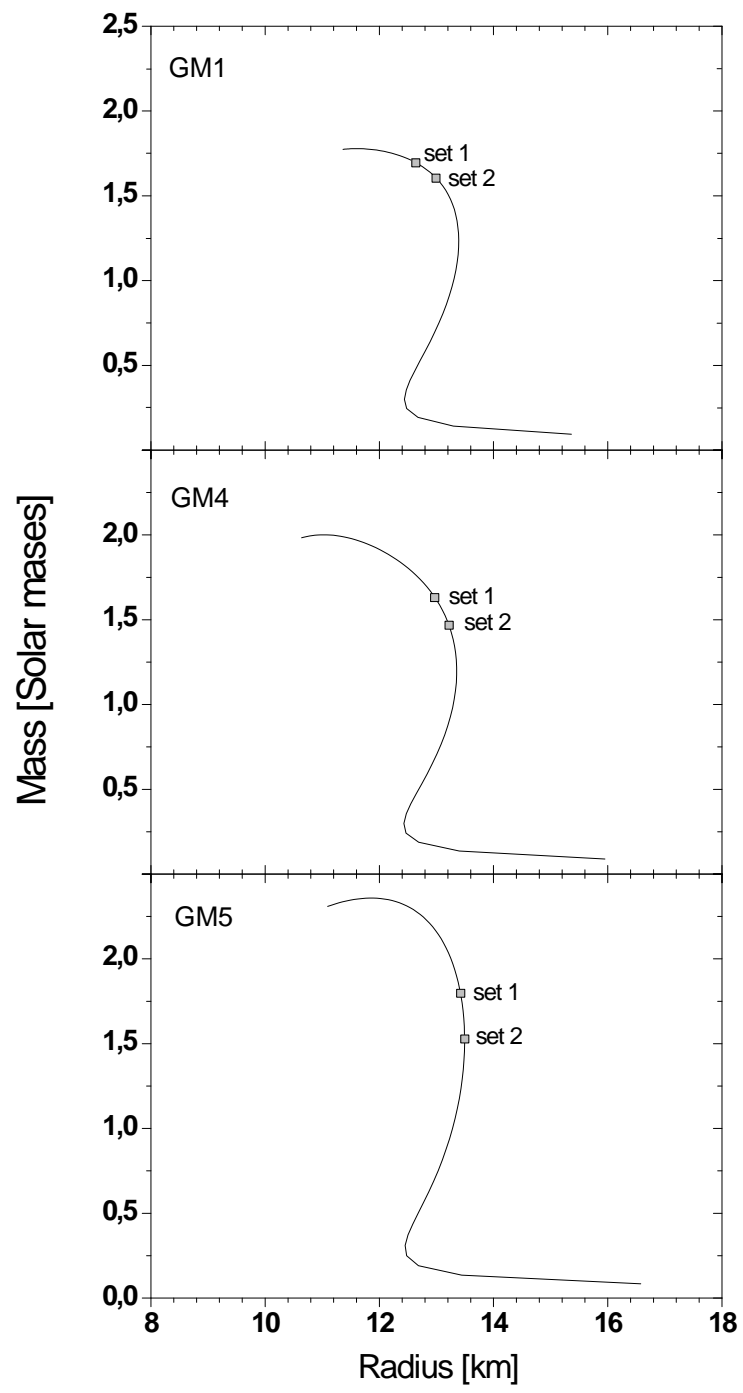

FIG. 2: Mass-radius relationship for hadronic stars at zero temperature with the GM1, GM4 and GM5 EOS. For a given parametrization of the NJL model, stars with a mass above the corresponding point given in the figure are hybrid stars containing quark cores. The mass-radius relationship for these hybrid stars is not shown in this figure.

transition to quark matter. The results are shown in Fig. 2 for neutron stars at zero temperature where we show the mass-radius relationship for hadronic stars and indicate the critical mass for the two selected parameterizations of the NJL model.

In the first seconds after their formation in a core collapse supernova explosion, neutron stars may have temperatures up to $\sim 50 \mathrm{MeV}$ and a large amount of trapped neutrinos. Thus, the results presented in Fig. 1 are not appropriate for the analysis of the critical mass in proto-neutron star conditions since they do not include the effect of trapped neutrinos. However, trapped neutrinos increase considerably the critical density $\rho_{c r}$ for the transition to quark matter [3]. Thus, it is possible that the transition is strongly inhibited in the initial moments of the evolution of neutron stars [4]. About one minute after its birth, there are almost no more trapped neutrinos in neutron star matter, while the temperature is still high (up to $\sim 10 \mathrm{MeV}[21]$ ). Thus, the results presented in Fig. 11 are valid for neutron stars older than $\sim 1$ minute. On the other hand, as apparent from Fig. 1 there is little variation in the critical Gibbs free energy per barion for temperatures below $\sim 10 \mathrm{MeV}$. Thus, the critical masses presented in Fig. 2 are also valid for neutron stars older than $\sim 1$ minute (in spite of being calculated considering hadronic matter at zero temperature). 


\section{DISCUSSION AND CONCLUSIONS}

In this paper we have analyzed the deconfinement transition from hadronic matter to quark matter and investigated the role of color superconductivity within the Nambu-Jona-Lasinio model. The study presented here is relevant for neutron stars older than $\sim 1$ minute, when there are almost no more trapped neutrinos in neutron star matter.

For the hadronic phase we have used three different parameterizations of a non-linear Walecka model which includes the whole baryon octet and electrons. One of them is the standard parametrization GM1 given by GlendenningMoszkowski [9]. This parametrization employs "low" values for the relative meson-hyperon couplings $x_{\sigma}, x_{\omega}$ and $x_{\rho}$. Larger values of these couplings make the EOS stiffer and, as shown in Table I, increase the value of the maximum mass of hadronic stars to values above $2 M_{\odot}$. These large values may be relevant in connection with recent measurements of highly-massive neutron stars which give one of the most stringent test on the overall stiffness of dense matter EOS. However, such measurements still have to be taken with caution (see discussion given in [22]). For example, the mass of the pulsar J0751+1807 was corrected from $M=(2.1 \pm 0.1) M_{\odot}[23]$ to $(1.26 \pm 0.14) M_{\odot}$ as new data became available [24]. There is also a series of measurements of extremely massive pulsars in globular clusters, where just the periastron advance has been determined but not the inclination angle of the orbit [25, 26, 27]. For the pulsar PSR J1748-2021B a mass of $(2.74 \pm 0.21) M_{\odot}$ is reported by using a statistical analysis for the inclination angle [26]. Also, recent measurements of post-Keplerian orbital parameters in relativistic binary systems containing millisecond pulsars give evidence for the existence of highly-massive compact stars. For example, the compact star associated to the millisecond pulsar PSR B1516+02B in the Globular Cluster NGC 5904 (M5) has a mass $M=1.94_{-0.19}^{+0.17} M_{\odot}(1 \sigma)$ [27]. Other constraints for the mass and radius have been obtained studying redshifted spectral lines extracted in the aftermath of an X-ray burst of the Low Mass X-ray Binary EXO 0748-676 in 2000 [28]. A model analysis of the $\mathrm{X}$-ray burst led to rather tight constraints for the mass and radius of the compact star of $M \geq(2.10 \pm 0.28) M_{\odot}$ and $R \geq(13.8 \pm 1.8) \mathrm{km}$ [29] which are based on the redshift measurement of [28]. However, a detailed multiwavelength analysis concluded that the mass of the compact star is more compatible with $1.35 M_{\odot}$ than with $2.1 M_{\odot}[30$. Moreover, follow-up observations of another burst in 2003 [31] could not confirm the spectral features seen in the burst spectra presented in [28]. While most of these measurements would need further confirmation, it is worth also exploring hadronic models that can produce stellar configurations with masses above $2 M_{\odot}$ (see the parameterizations GM4 and GM5 in Table I).

Employing the results of Fig. 1 and integrating the Tolman-Oppenheimer-Volkoff equations of relativistic stellar structure for the hadronic EOSs given in Table I we have calculated the critical compact star mass $M_{c r}$ above which they should contain a quark core. Pure hadronic stars with $M_{h}>M_{c r}$ are very unlikely to exist, while pure hadronic stars with $M_{h}<M_{c r}$ are safe with respect to a sudden transition to quark matter. Notice that the critical mass is defined here in a different way as in Refs. [5, 7, 32]. In the here-presented bulk analysis, the transition begins when the Gibbs conditions $\Delta P \equiv P_{h}-P_{q}=0$ and $\Delta g \equiv g_{h}-g_{q}=0$ are verified at a given temperature. However, for the nucleation of finite size bubbles, it is necessary to have an overpressure $\Delta P>0$ with respect to the bulk transition point due to surface and curvature effects. For a given overpressure there is a probability (and a corresponding nucleation time) to nucleate a quark bubble due to quantum or thermal fluctuations. In [5, 7] the critical mass was defined as the value of the gravitational mass of a hadronic star for which the nucleation time due to quantum fluctuations is equal to one year. Calculations of the critical mass done within the frame of the MIT bag model show that surface effects are strong for values of the Bag constant $B$ smaller than $\sim 100 \mathrm{MeV} \mathrm{fm}^{-3}[7]$. That is, within the MIT bag model, the effect of the surface tension $\sigma$ is strong for strange stars but it is small if the parameters of the EOS correspond to hybrid stars. For the parameterizations of the NJL model employed in this work only hybrid stars are allowed. Thus, finite size effects are not expected to introduce qualitative modifications in our results.

Employing set 1 for the Nambu-Jona-Lasinio model (see Table II) we find that there are not large variations in the critical mass for the three different parameterizations of the hadronic matter equation of state. As seen in Fig. 2 the critical mass is in the range 1.65-1.80 $M_{\odot}$ for set 1 , which is not a large difference if we consider the larger variation in the maximum mass of hadronic stars within the three parameterizations $\left(M_{\max }\right.$ between 1.78 and $2.35 M_{\odot}$, see Table I). A similar result is found employing set 2 for the Nambu-Jona-Lasinio model, i.e. the critical mass is in the range 1.45-1.60 $M_{\odot}$ (see Fig. 2). This is in coincidence with previous results obtained for hybrid stars within the MIT Bag model [7]. As mentioned above, in the case of the MIT Bag model stars containing quark phases are strange stars for low values of the Bag constant $B$, and hybrid stars for sufficiently large values of $B$. For the values of $B$ corresponding to strange stars the critical mass may vary essentially from zero to near the maximum mass of hadronic stars, depending on the value of other parameters such as the superconducting gap $\Delta$ and the surface tension $\sigma$. However, for the values of $B$ corresponding to hybrid stars the critical mass is always close to (but smaller than) the maximum mass of hadronic stars, rather independently of the value of other parameters [7]. The parameterizations employed here of the Nambu-Jona-Lasinio model allow only for the existence of hybrid stars and show the same characteristics of the critical mass, allowing the existence of a mixed population of compact stars (pure hadronic up to the critical mass and hybrid above the critical mass). 


\section{ACKNOWLEDGEMENTS}

This work was supported in part by CONICET (Argentina) grant \# PIP 6084 and by ANPCyT (Argentina) grants \# PICT04 03-25374 and \# PICT07 03-00818.

[1] K. Iida and K. Sato, Phys. Rev. D 58, 2538 (1998).

[2] M. L. Olesen and J. Madsen, Phys. Rev. D 49, 2698 (1994).

[3] G. Lugones and O. G. Benvenuto, Phys. Rev. D 58, 083001 (1998).

[4] O. G. Benvenuto and G. Lugones, Mon. Not. R.A.S. 304, L25 (1999).

[5] I. Bombaci, I. Parenti, I. Vidaña, Astrophys.J. 614, 314 (2004).

[6] G. Lugones and I. Bombaci, Phys. Rev. D 72, 065021 (2005).

[7] I. Bombaci, G. Lugones, I. Vidaña, Astronomy and Astrophysics 462, 1017 (2007).

[8] Z. Fodor and S.D. Katz, JHEP 0404, 050 (2004).

[9] N.K. Glendenning and S.A. Moszkowski, Phys. Rev. Lett. 67, 2414 (1991).

[10] J.D. Walecka, Ann. Phys. 83, 491 (1974); B.D. Serot and J.D. Walecka, Adv. Nucl. Phys. 16, 1 (1986).

[11] D.P. Menezes and C. Providência, Phys. Rev. C 68, 035804 (2003); A.M.S. Santos and D.P. Menezes, Phys. Rev. C 69, $045803(2004)$.

[12] M. Huang, P. f. Zhuang and W. q. Chao, Phys. Rev. D 67, 065015 (2003).

[13] S. B. Ruester, V. Werth, M. Buballa, I. A. Shovkovy and D. H. Rischke, Phys. Rev. D 72, 034004 (2005).

[14] D. Blaschke, S. Fredriksson, H. Grigorian, A. M. Oztas and F. Sandin, Phys. Rev. D 72, 065020 (2005).

[15] P. Rehberg, S. P. Klevansky and J. Hufner, Phys. Rev. C 53, 410 (1996).

[16] T. Hatsuda and T. Kunihiro, Phys. Rept. 247, 221 (1994).

[17] M. Buballa, Phys. Rept. 407, 205 (2005).

[18] E. Farhi and R.L. Jaffe, Phys. Rev. D 30, 2379 (1984).

[19] G. Lugones and J. E. Horvath, Phys. Rev. D 66, 074017 (2002).

[20] G. Lugones and J. E. Horvath, Astronomy and Astrophysics 403, 173 (2003).

[21] J. A. Pons et al., Astrophys. J. 513, 780, (1999).

[22] J. Aichelin and J. Schaffner-Bielich, arXiv:0812.1341 v2 [nucl-th].

[23] D. J. Nice, E. M. Splaver, I. H. Stairs, O. Löhmer, A. Jessner, M. Kramer and J. M. Cordes, Astrophys. J. 634, 1242 (2005).

[24] D. J. Nice, I. H. Stairs and L. E. Kasian, AIP Conference Proceedings 983, 453 (2008).

[25] S. M. Ransom, J. W. T. Hessels, I. H. Stairs, P. C. C. Freire, F. Camilo, V. M. Kaspi and D. L. Kaplan, Science 307, 892 (2005).

[26] P. C. C. Freire, S. M. Ransom, S. Bégin, I. H. Stairs, J. W. T. Hessels, L. H. Frey and F. Camilo, Astrophys. J. 675, 670 (2008).

[27] P. C. C. Freire, A. Wolszczan, M. van den Berg and J. W. T. Hessels, Astrophys. J. 679, 1433 (2008).

[28] J. Cottam, F. Paerels and M. Mendez, Nature 420, 51 (2002).

[29] F. Özel, Nature 441, 1115 (2006).

[30] K. J. Pearson, R. Hynes, D. Steeghs, P. Jonker, C. Haswell, A. King, K. O’Brien, G. Nelemans and M. Mendez, Astrophys. J. 648, 1169 (2006).

[31] J. Cottam, F. Paerels, M. Mendez, L. Boirin, W. H. G. Lewin, E. Kuulkers and J. M. Miller, Astrophys. J. 672, 504 (2008).

[32] I. Bombaci, P. K. Panda, C. Providencia and I. Vidana, Phys. Rev. D 77, 083002 (2008). 